\section{Visual performance of acrylic and PMMA intraocular lenses}

\begin{abstract}
Aim To evaluate the quality of visual functions after cataract surgery and intraocular lens (IOL) implantation with different lens materials and compare the results with agematched subjects with clear phakic eyes. Methods Control and pseudophakic groups involved individuals aged between 50 and 75 years, without any accompanying ocular or systemic disease. In all, 50 eyes implanted with foldable acrylic IOLs, and 41 eyes implanted with polymethyl-methacrylate (PMMA) IOLs were compared with 45 phakic eyes as controls. Visual functions were evaluated for contrast sensitivity function and glare disability. The results were compared statistically using one-way analysis of variance (ANOVA).

Results At high luminance levels, the difference among groups for contrast sensitivity was statistically significant for all spatial frequencies $(P<0.05)$. Although the acrylic IOL-implanted eyes had better results at all three spatial frequencies, no significant difference existed between the two study groups for these spatial frequencies $(P>0.05)$. Glare disability scores were significantly higher in the PMMA-IOL group compared to the control and acrylic-IOL groups.

Conclusion The visual quality achieved in pseudophakic eyes was not as good as in clear phakic eyes in regard to contrast sensitivity and glare. However, acrylic IOLs fared better than PMMA IOLs.

Eye (2003) 17, 238-242. doi:10.1038/

sj.eye. 6700290
\end{abstract}

Cataract and Refractive Surgery Service Department of Ophthalmology Istanbul University Istanbul Faculty of Medicine Istanbul, Turkey

Correspondence: R Altan-Yaycioglu Ataturk Cad Behice Yazgan Apt No. 33, D: 501120 Adana Turkey

Tel: 0090532373 5585: Fax: 00903223271273 E-mail: raltanya@ yahoo.com

This study has been presented as a poster at SOE '99, XIlth European Congress of Ophthalmology, Stockholm, Sweden, June 27-July 1, 1999
Keywords: pseudophakia; polymethyl-methacrylate; acrylic intraocular lens; visual quality; contrast sensitivity function; glare disability

\section{Introduction}

Phacoemulsification with intraocular lens (IOL) implantation is considered to be one of the most successful interventions in modern medicine.
N Gozum, E Safgonul Unal, R Altan-Yaycioglu, A Gucukoglu and C Ozgun

The incisions getting smaller together with the development of better IOL materials and designs provide the best possible visual rehabilitation for patients. Nowadays surgery is recommended for cases with early cataracts as well. The reported results and high benefit-torisk ratio of cataract surgery encourage patients for early surgery and make them place their expectations at a very high level. They expect the same visual quality as clear phakic eyes.

Excellent visual acuity results of the IOLs obtained with Snellen charts reflect the visual function at $100 \%$ contrast levels. However, reallife situations are associated with various levels of illumination and objects with different shades and contrasts. Contrast sensitivity and glare disability testing are useful methods for evaluating the problems of patients in daily

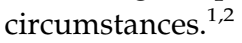

This study was undertaken to evaluate the visual performance of pseudophakic subjects with acrylic and PMMA IOLs through contrast sensitivity and glare disability testing and to compare the results with clear phakic eyes.

Materials and methods

In this prospective clinical study we enrolled consecutive patients, who were aged between 50 and 75 years, status post uneventful phacoemulsification 6 months ago, diagnosed with senile cataract and implanted with posterior chamber IOLs. Eyes with a history of intraoperative posterior capsular rupture and vitreous loss, postoperative intraocular pressure rise requiring medical or surgical treatment, pupil deformities such as synechia or capture, decentred IOLs, intensive pigment deposits on corneal endothelium or IOL surface, pupillary membranes, macular disease - cystoid macular oedema or macular degeneration - vascular occlusions, retinal detachment or eyes with neodymium:YAG laser capsulotomies were excluded from the study. Patients with moderate or severe PCO or residual plaques 
were excluded from the study to eliminate the effects of PCO to the contrast sensitivity or glare, and only eyes with mild PCO were included in the study. All subjects gave informed consent prior to testing.

Patients were divided into two groups. The first group consisted of 41 patients who were implanted with $13.5 \mathrm{~mm}$ polymethyl-methacrylate (PMMA) IOL, with $6.0 \mathrm{~mm}$ optic (Alcon Labs, Fort Worth, TX, USA) after performing phacoemulsification through a scleral tunnel incision. The second group consisted of 50 patients who were implanted with $13.0 \mathrm{~mm}$ acrylic IOL with $6.0 \mathrm{~mm}$ optic (Acrysof, MA60BA, Alcon Labs, Fort Worth, TX, USA) after performing phacoemulsification through a $3.5 \mathrm{~mm}$ temporal clear corneal incision.

In all, 45 subjects with phakic eyes were recruited from the outpatient clinic as controls. To be included in the control group, the subjects had to fulfil the following criteria: (1) age between 50 and 74 years, (2) best corrected visual acuity of 20/20, (3) clear or sclerotic lens, clear cornea, regular pupil, and (4) no past or present ocular disease.

Following the standardized eye examination including best spectacle corrected visual acuity (BSCVA), slit lamp examination, applanation tonometry, and dilated examination of the lens and fundus, patients underwent contrast sensitivity function and glare disability tests with the full refractive correction and natural (undilated) pupils. The time elapsed from surgery was 6 months for pseudophakic patients.

Contrast sensitivity function was measured with best correction using the Gradual Contrast Sensitivity Test Chart (Opsia Laboratories, Saint Agne, France). This is a rear-illuminated chart bearing optotypes on columns with decreasing contrast from left to right and decreasing size from top to bottom. The size of the letters on the top line correspond to Snellen letters of 20/400 visual acuity and the bottom line to $20 / 20$. The viewing distance was $3 \mathrm{~m}$. Letters are grouped into three spatial frequencies as low, medium and high. Letters corresponding to a visual acuity of up to $20 / 100$ are in the low spatial frequency group, those between $20 / 80$ and $20 / 40$ are in the medium, spatial frequency group, and those between $20 / 30$ and $20 / 20$ are in the high spatial frequency group. Luminance of the screen can be varied as high photopic $\left(700 \mathrm{~cd} / \mathrm{m}^{2}\right)$, mesopic $\left(85 \mathrm{~cd} / \mathrm{m}^{2}\right)$, and low photopic $\left(5 \mathrm{~cd} / \mathrm{m}^{2}\right)$. The test was performed in a standardized room light $(50 \mathrm{~L})$ for high photopic and mesopic illumination and in a dark room for low photopic illumination. The patients were asked to read a line corresponding to each spatial frequency. One point was given to each letter read correctly on the line, and this gave the contrast sensitivity function for that spatial frequency. The test was repeated for three luminance levels.
To measure the glare disability, the glare test in the program of Computerized Autorefractometer Model 560 (Humphrey, Allergan, San Leandro, CA, USA) was used. Patients were asked to read from the low contrast card after entering their refraction error. This card includes Snellen letter optotypes between 0.1 and 0.8 Snellen visual acuity. The lowest correctly read letter line was recorded as low contrast visual acuity. Then the test was repeated under glare light sources and the line read correctly was recorded as the low contrast visual acuity under glare source. The difference between the two values was calculated as the glare disability score. The illumination of the low contrast card was $47 \mathrm{ft} \mathrm{L}$ and that of the glare source $5000 \mathrm{ft}$.

For statistical analyses, Graphped INSTAT V2.02 software was used. Three groups were compared for contrast sensitivity and glare disability using a one-way analysis of variance (ANOVA).

\section{Results}

The age range in groups with the mean and standard deviation are shown in Table 1. The age of the patients in the groups was not significantly different $(P>0.05)$. The difference for the BSCVA also was not significant among the groups $(P>0.05)$ (Table 1$)$. In six $(12 \%)$ of the acrylic IOL-implanted eyes, and in eight (19.5\%) of the PMMA implanted eyes, mild posterior capsular opasification (PCO) developed at 6 months. The difference between two study groups for PCO rates was statistically insignificant $(P>0.05)$.

The results of the mean contrast sensitivity testing for three luminance levels are shown in Tables 2-4. Mean contrast sensitivity decreased as luminance decreased and spatial frequency increased in all groups. At high luminance levels, the difference among the groups was statistically significant for all spatial frequencies $(P<0.05)$. The scores of the control group were significantly higher than both of the acrylic and the PMMA groups. While the scores of the acrylic and PMMA groups did not differ significantly for low and medium spatial frequencies $(P>0.05)$, the scores of the

Table 1 Demographic data of patients in the acrylic or PMMA IOL-implanted groups and with normal phakic eyes as the control group

\begin{tabular}{lclll}
\hline Groups & $n^{\mathrm{a}}$ & $\begin{array}{l}\text { Age range } \\
\text { (years) }\end{array}$ & $\begin{array}{l}\text { Mean age } \pm S D \\
\text { (years) }\end{array}$ & BSCVA $^{\mathrm{b}}$ \\
\hline Acrylic & 50 & $50-75$ & $62.4 \pm 9.0$ & $0.99 \pm 0.02$ \\
PMMA & 41 & $50-75$ & $62.3 \pm 7.9$ & $0.99 \pm 0.03$ \\
Control & 45 & $50-74$ & $59.4 \pm 6.3$ & $1.00 \pm 0.00$ \\
\hline
\end{tabular}

${ }^{a} n$ : number of patients.

bBSCVA: best spectacle corrected visual acuity. 
Table 2 Mean contrast sensitivity scores of patients with acrylic or PMMA IOL-implanted eyes or clear phakic eyes at high photopic luminance $\left(700 \mathrm{~cd} / \mathrm{m}^{2}\right)$

\begin{tabular}{llll}
\hline Groups & Low SF & Medium SF & High SF \\
\hline Acrylic & $9.21 \pm 0.90$ & $7.65 \pm 1.62$ & $5.77 \pm 2.15$ \\
PMMA & $9.02 \pm 1.33$ & $7.29 \pm 2.03$ & $4.80 \pm 2.43$ \\
Control & $9.87 \pm 0.34$ & $9.02 \pm 0.69$ & $6.93 \pm 1.67$ \\
-value $^{\text {b }}$ & $<0.05^{*}$ & $<0.05^{*}$ & $<0.05^{*}$ \\
\hline
\end{tabular}

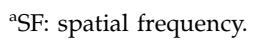

${ }^{b} P$ : probability index $\left(P>0.05\right.$ : statistically insignificant, $P<0.05^{*}$ : statistically significant with ANOVA).

Table 3 Mean contrast sensitivity scores of patients with acrylic or PMMA IOL-implanted eyes or clear phakic eyes at mesopic luminance $\left(85 \mathrm{~cd} / \mathrm{m}^{2}\right)$

\begin{tabular}{lccc}
\hline Groups & Low SF & Medium SF & High SF \\
\hline Acrylic & $9.38 \pm 0.89$ & $7.15 \pm 1.65$ & $4.88 \pm 2.06$ \\
PMMA & $9.00 \pm 1.38$ & $6.51 \pm 2.28$ & $4.10 \pm 2.41$ \\
Control & $9.84 \pm 0.42$ & $8.22 \pm 1.20$ & $5.18 \pm 2.00$ \\
-value $^{\mathrm{b}}$ & $<0.05^{*}$ & $<0.05^{*}$ & $>0.05$
\end{tabular}

${ }^{\text {aSF: }}$ spatial frequency.

b $P$ : probability index $\left(P>0.05\right.$ : statistically insignificant; $P<0.05^{*}$ : statistically significant with ANOVA).

Table 4 Mean contrast sensitivity scores of patients with acrylic or PMMA IOL-implanted eyes or clear phakic eyes at low photopic luminance $\left(5 \mathrm{~cd} / \mathrm{m}^{2}\right)$

\begin{tabular}{llcc}
\hline Groups & Low SF & Medium SF & High SF \\
\hline Acrylic & $7.94 \pm 1.26$ & $3.81 \pm 1.51$ & $1.39 \pm 1.00$ \\
PMMA & $7.02 \pm 1.78$ & $3.37 \pm 1.61$ & $1.00 \pm 1.10$ \\
Control & $9.22 \pm 0.95$ & $4.64 \pm 1.37$ & $1.53 \pm 1.38$ \\
& & & $>0.05$
\end{tabular}

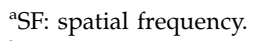

${ }^{\mathrm{b}} P$ : probability index $\left(P>0.05\right.$ : statistically insignificant, $P<0.05^{*}$ : statistically significant with ANOVA).

acrylic group were statistically higher than those of the PMMA group for high spatial frequency $(P<0.05)$.

At mesopic and low photopic conditions, the differences among groups were statistically significant for low and medium spatial frequencies $(P<0.05)$, but insignificant for high spatial frequency $(P>0.05)$. At mesopic conditions, scores of the control group were significantly higher than those of the study groups at low and medium spatial frequencies $(P<0.05)$. Although the acrylic IOL implanted eyes had better results at all three spatial frequencies, no significant difference existed between the two study groups for these spatial frequencies $(P>0.05)$.

At low luminance levels, the scores of the control group were significantly higher than those of the study
Table 5 Mean glare disability scores of patients with acrylic or PMMA IOL-implanted eyes or clear phakic eyes

\begin{tabular}{lc}
\hline Group & Mean glare disability score \\
\hline Acrylic & $1.12 \pm 0.87$ \\
PMMA & $1.90 \pm 1.70$ \\
Control & $0.64 \pm 1.09$ \\
& $<0.05^{*}$ \\
$P$-value & \\
\hline${ }^{a} P$ : probability index $\left(P>0.05:\right.$ statistically insignificant; $P<0.05^{*}$ : statisti- \\
cally significant with ANOVA).
\end{tabular}

groups at low and medium spatial frequencies $(P<0.05)$. The scores of the acrylic group were significantly higher than the PMMA group for low spatial frequency $(P<0.05)$, but did not differ for high spatial frequency $(P>0.05)$.

Table 5 shows the mean glare disability scores. Glare disability was lowest in the control group. The results of the control and acrylic groups were not significantly different $(P>0.05)$. On the other hand, the differences between the control group and the PMMA group and between the acrylic and PMMA scores were statistically significant $(P<0.05)$

\section{Disscussion}

PMMA was the first material used in the manufacture of IOLs; ${ }^{3}$ however, the introduction of phacoemulsification has prompted the use of foldable lenses to be inserted through smaller wounds. All these lenses have proved themselves to be nontoxic, highly biocompatible, and stable. ${ }^{4-6}$

Many studies have reported excellent visual acuity results with all IOL types. ${ }^{7,8}$ Likewise, both groups in our study had very high levels of BSCVA and did not differ significantly from age-matched phakic eyes with clear lenses $(P>0.05)$. However, with the Snellen chart it is only possible to evaluate the visual acuity and identify details under 100\% contrast level. More sophisticated methods of evaluating visual functions like contrast sensitivity and glare disability testing help explain the difficulties encountered by patients in everyday life. ${ }^{1,2,9}$ In order to detect and discriminate larger objects in our environment, spatial contrast sensitivity testing was suggested as a useful method..$^{10}$ For this purpose, the visual functions of normal senile people, who had uncomplicated phacoemulsification procedures followed by in-the-bag implanted PMMA or acrylic IOLs using the contrast sensitivity and glare disability testing, were compared in the present study.

The ages of patients ranged between 50 and 75 years in the control and study groups, and there was no statistically significant difference in age between the groups $(P>0.05)$. Since contrast sensitivity function and 
glare disability testing might be affected by the age of the patient, the size of the pupil, and many ophthalmological or systemic diseases, ${ }^{2,9,11,12}$ patients who had irregular or out of the range of $3-5 \mathrm{~mm}$ pupils, or with any other systemic or ophthalmologic disease were excluded from the study.

It is previously reported that early cataracts cause loss of contrast sensitivity and increase in glare disability. ${ }^{13}$ The purpose of this study was not to compare the preand postoperative differences. Adamsons et al ${ }^{14}$ compared the subjective and objective visual functions. They found that the preoperative visual functions were positively correlated with postoperative objective visual functions by means of visual acuity and contrast sensitivity.

To our knowledge, there is no other report using the opsia gradual sensitivity chart. Our test chart resembles Regan charts, in that the test is being performed at $3 \mathrm{~m}$ and using letters instead of sinusoidal gratings, lowering in size and contrast of the letters. It is reported that letters are more reliable compared to sinewave grating charts. ${ }^{15}$ Actual spatial frequency depends on the spatial frequency content of the real-world target. ${ }^{10}$ Decrease of contrast sensitivity at low and medium spatial frequencies causes difficulties in recognizing human faces, road signs, houses and reading. ${ }^{10}$ Utilization of foldable IOLs has led to their comparison with PMMA lenses, of which the optic qualities have been proved in the past. Pseudophakic subjects with PMMA IOLs are reported to have less contrast sensitivity than phakic subjects in each frequency, being more pronounced in high spatial frequency. ${ }^{16,17}$ In contrast, Furuskog and Nilsson ${ }^{18}$ reported both groups as having similar results for contrast sensitivity. In this study, pseudophakic patients with PMMA lenses had lower contrast sensitivity scores compared to the ones with acrylic IOL and to the control group.

In another study both in-the-bag implanted PMMA and silicone IOLs were compared, and although insignificant, silicone lenses were found to have lower values in low contrast visual acuity and with a glare source $(P>0.05){ }^{19}$

Skorpik et al compared in-the-bag implanted PMMA IOLs with sulcus implanted silicone IOLs and found no statistically significant difference in any luminance level under mesopic conditions. ${ }^{19,20}$ After the introduction of foldable acrylic lenses, Kohnen $e t$ al ${ }^{8}$ compared the visual functions of various IOLs, and the difference between PMMA and acrylic intraocular lenses was found to be statistically insignificant for contrast sensitivity.

The present study differs from previously reported studies in that contrast sensitivity was tested for different spatial frequencies at different illumination levels. In this study, it is shown that the scores decreased with the decrease in illumination and increase in spatial frequency. The highest values were obtained for the phakic control group followed by the acrylic group. Although this difference was not significant for low spatial frequency at high photopic conditions and for high spatial frequency at mesopic and low photopic conditions $(P>0.05)$, phakic eyes had significantly better visual functions than pseudophakic eyes $(P<0.05)$. At mesopic conditions, which represent illumination in cloudy weather, the values of the acrylic group were approaching those of phakic eyes at low spatial frequency and the difference was insignificant $(P>0.05)$.

Light scatter within the eye causes disability glare, a contrast lowering effect. The computerized autorefractometer measures visual acuity impairment in the presence of glare with low contrast letters. In a study, Beckman and co-workers used a similar device (Humphrey Automatic Refractor Model 570) to measure the impairment of visual acuity for low contrast optotypes as a result of glare for subjects with normal and cataractous eyes. They compared the results with subjective glare and intraocular light scatter, and found no correlation with subjective glare and very weak correlation with intraocular light scatter. ${ }^{21}$ Masket $^{22}$ compared the postoperative disability glare with the preoperative values and found an improvement. However, glare disability is reported to be higher in patients with posterior chamber intraocular lenses than in normal controls. ${ }^{23}$

In the presence of a glare source, phakic eyes had less decrease in visual acuity $(P<0.05)$. Although not as good as the phakic group, the results in the acrylic group were significantly better than the PMMA group $(P<0.05)$. In the present study, $\mathrm{PCO}$ was more common in the PMMA IOL-implanted group, however, the difference was statistically insignificant $(P>0.05)$. Similarly, acrylic lenses are reported to result in PCO, although less frequent than PMMA lenses, with a significant difference. ${ }^{24}$ Although the difference for mild PCO was insignificant between the PMMA and acrylic IOL groups in this study, the high glare disability and contrast sensitivity in the PMMA group might be the result of this insignificant high incidence of mild PCOs. Nadler $\mathrm{et}_{\mathrm{al}} \mathrm{l}^{23}$ also suggested a correlation between the amount of $\mathrm{PCO}$ and glare score.

In conclusion, although none of the IOL materials in this study reached the visual quality of clear phakic eyes, acrylic IOLs gave the second best results in contrast sensitivity and glare disability testing after clear lenses. We have the opinion that in addition to small corneal incision, less astigmatism, and faster rehabilitation, less disability glare and better contrast sensitivity are other advantages of foldable acrylic lenses. However, they 
need to be compared with other types of foldable lenses in regard to visual qualities.

\section{Acknowledgements}

This study was supported by Istanbul University Research Found BEKADEP.

The authors have no financial interest in any of the materials used in this study.

\section{References}

1 Jindra LF, Zemon V. Contrast sensitivity testing: a more complete assessment of vision. J Cataract Refract Surg 1989; 15: $141-148$

2 Wollner I. Detention of visual defects using the contrast sensitivity function. Int Ophthalmol Clin 1980; 20: 135-153.

3 Stamper RL, Sugar A, Ripkin DJ. Historical introduction. In: Intraocular Lenses. Basic and Clinical Applications. American Academy of Ophthalmology: San Francisco, 1993, 1-5.

4 Fogle JA, Blaydes JE, Fritz KJ, Blaydes SH, Mazzocco TR, Peiffer RL, Cook C, Wright E. Clinicopathologic observations of a silicone posterior chamber lens in a primate model. J Cataract Refract Surg 1986; 12: 281-284.

5 Cook C, Peiffer RL, Mazzocco TR. Clinical and pathologic evaluation of a flexible silicone posterior chamber lens design in a rabbit model. J Cataract Refract Surg 1986; 12: 130-134.

6 Packard RB, Garner A, Arnott EJ. Poly-HEMA as a material for intraocular lens implantation: a preliminary report. Br J Ophthalmol 1981; 65: 585-587.

7 Johansen J, Dam-Johansen M, Olsen T. Contrast sensitivity with silicone and poly (methyl methacrylate) intraocular lenses. J Cataract Refract Surg 1997; 23: 1085-1088.

8 Kohnen S, Ferrer A, Brauweiler P. Visual function in pseudophakic eyes with poly (methyl methacrylate), silicone, and acrylic intraocular lenses. J Cataract Refract Surg 1996; 22: 1303-1307.

9 Masket S. Glare disability and contrast sensitivity function in the evaluation of symptomatic cataract. In: Stamper RL, Obstbaum SA (eds). Ophthalmology Clinics of North America. WB Saunders: Philadelphia, 1991, pp. 365-381.

10 Owsley C, Sloane ME. Contrast sensitivity, acuity, and the perception of 'real-world' targets. Br J Ophthalmol 1987; 71: 791-796.
11 Rubin GS, West SK, Munoz B, Bondeen-Roche K, Zeger S, Schein O, Fried LP. A comprehensive assessment of visual impairment in a population of older Americans: the SEE study. Salisbury Eye Evaluation Project. Invest Ophthalmol Vis Sci 1997; 38: 557-568.

12 Arden GB, Gucukoglu A. Grating test of contrast sensitivity in patients with retrobulbar neuritis. Arch Ophthalmol 1978; 96: 1626-1629.

13 Rubin GS, Adamsons IA, Stark WJ. Comparison of acuity, contrast sensitivity, and disability glare before and after cataract surgery. Arch Ophthalmol 1993; 111: 56-61.

14 Adamsons IA, Vitale S, Stark WJ, Rubin GS. The association of postoperative subjective visual function with acuity, glare, and contrast sensitivity in patients with early cataract. Arch Ophthalmol 1996; 114: 529-536.

15 Rubin GS. Reliability and sensitivity of clinical contrast sensitivity tests. Clin Vis Sci 1988; 2: 169-177.

16 Gozum N, Yildirim A, Gucukoglu A. Contrast sensitivity glare and testing in pseudophakia and early cataracts. In: Sveges I, Follmann P, Manduzzu CD (eds). SOE'97 XIth Congress European Society of the Ophthalmology. Bologna: London, 1997, pp. 415-417.

17 Pfoff DS, Werner JS. Effect of cataract surgery on contrast sensitivity and glare in patients with 20/50 or better Snellen acuity. J Cataract Refract Surg 1994; 20: 620-625.

18 Furuskog P, Nilsson BY. Contrast sensitivity in patients with posterior chamber intraocular lens implants. Acta Ophthalmol (Copenh) 1988; 66: 438-444.

19 Knorz MC, Lang A, Hsia T, Poepel B, Seiberth V, Liesenhoff H. Comparison of the optical and visual quality of poly(methyl methacrylate) and silicone intraocular lenses. J Cataract Refract Surg 1993; 19: 766-771.

20 Skorpik C, Gottlob I, Weghaupt H. Comparison of contrast sensitivity between posterior chamber lenses of silicone and PMMA material. Graefe's Arch Clin Exp Ophthalmol 1989; 227: 413-416.

21 Beckman C, Scott R, Garner LF. Comparison of three methods of evaluating glare. Acta Ophthalmol Copenh 1992; 70: 53-59.

22 Masket S. Reversal of glare disability after cataract surgery. J Cataract Refract Surg 1989; 15: 165-168.

23 Nadler DJ, Jaffe NS, Clayman HM, Jaffe MS, and Luscombe SM. Glare diability in eyes with intraocular lenses. Am J Ophthalmol 1984; 97: 43-47.

24 Ursell PG, Spalton DJ, Pande MV, Hollick EJ, Barman S, Boyce J, Tilling K. Relationship between intraocular lens biomaterials and posterior capsule opacification. J Cataract Refract Surg 1998; 24: 352-360. 